\title{
Tuning the metamagnetism of an antiferromagnetic metal
}

\author{
J. B. Staunton, ${ }^{1}$ M. dos Santos Dias, ${ }^{1,2}$ J. Peace, ${ }^{1}$ Z. Gercsi, ${ }^{3}$ and K. G. Sandeman ${ }^{3}$ \\ ${ }^{1}$ Department of Physics, University of Warwick, Coventry CV4 7AL, United Kingdom \\ ${ }^{2}$ Peter Grünberg Institut and Institute for Advanced Simulation, Forschungszentrum Jülich and JARA, D-52425 Jülich, Germany \\ ${ }^{3}$ Department of Physics, Blackett Laboratory, Imperial College London, London SW7 2AZ, United Kingdom
}

(Received 14 June 2012; revised manuscript received 14 September 2012; published 13 February 2013)

\begin{abstract}
We describe a "disordered local moment" first-principles electronic structure theory which demonstrates that tricritical metamagnetism can arise in an antiferromagnetic metal due to the dependence of local moment interactions on the magnetization state. Itinerant electrons can therefore play a defining role in metamagnetism in the absence of large magnetic anisotropy. Our model is used to accurately predict the temperature dependence of the metamagnetic critical fields in CoMnSi-based alloys, explaining the sensitivity of metamagnetism to Mn-Mn separations and compositional variations found previously. We thus provide a finite-temperature framework for modeling and predicting different metamagnets of interest in applications such as magnetic cooling.
\end{abstract}

DOI: 10.1103/PhysRevB.87.060404

PACS number(s): 75.30.Kz, 75.10.Lp, 75.50.Ee, 75.30.Sg

The application of a magnetic field to an antiferromagnet can cause abrupt changes to its magnetic state. ${ }^{1}$ While such metamagnetic transitions have been known for a long time in materials such as $\mathrm{FeCl}_{2}$ (Ref. 2) and $\mathrm{MnF}_{2},{ }^{3}$ it is their association with technologies such as magnetic cooling ${ }^{4}$ that has driven recent efforts to control metamagnetism in the room temperature range, and in accessible magnetic fields. Indeed, a large, or even "giant" magnetocaloric effect can arise at a first-order metamagnetic phase transition such as is found in FeRh. ${ }^{5}$ However, the thermomagnetic hysteresis associated with most first-order transitions results in significant inefficiency during magnetic cycling. Therefore, (tri)critical transitions that straddle first- and second-order behavior are of great interest. It is worth noting that tricritical points have also been examined in the context of other technologies, such as liquid crystal displays. ${ }^{6}$

Given the interest in finding room temperature magnetic refrigerants, a reexamination of tricritical antiferromagnetic (AFM) metamagnets is warranted. At present there are only two first-order magnetic refrigerants, La-Fe-Si (Ref. 7) and $\mathrm{MnFe}(\mathrm{P}, \mathrm{As}, \mathrm{Si}){ }^{8}$ at an advanced stage of deployment in prototype cooling devices. Both materials are ferromagnets (FMs) with field-induced metamagnetic critical points. However, tricriticality allows AFMs to emulate the low hysteresis, high entropy change properties of their FM cousins. For such real materials the greatest challenge is to understand the mixed localized/itinerant electron spin nature of their magnetism, and how this influences tricritical properties. Metamagnetic transitions, however, have previously been investigated in terms of either spin effect alone.

In AFM insulators, ${ }^{6}$ magnetic field-driven phase transitions can be understood qualitatively using a localized (classical) spin Hamiltonian with pairwise isotropic exchange interactions, a source of magnetic anisotropy, and a Zeeman external magnetic field term. For example, Nagamiya ${ }^{9}$ showed that, if the localized spins of a helical AFM are pinned by anisotropy and crystal field effects to spiral around a particular direction, the effect of a magnetic field brings about a first-order transition to a fan structure where the moments now oscillate about the field direction. At higher fields, the fan angle smoothly reduces to zero to establish a high magnetization phase at a second-order transition. However, if anisotropy effects are negligible, the helical order has no favored axis and, once a magnetic field is applied, the helix plane orients perpendicular to the field. With increasing field the moments cant smoothly into a conical spiral towards the field's direction with a second-order transition to a high magnetization phase. Thus, in this localized picture, first-order metamagnetism relies on a source of anisotropy.

Metamagnetism is accounted for differently in an itinerant electron system. Seminal work by Wohlfarth and Rhodes, ${ }^{10}$ Moriya and Usami, ${ }^{11}$ developed and extended by many others, e.g., Refs. 12 and 13, derived the coefficients of a LandauGinzburg free energy expansion for an AFM exposed to a uniform magnetic field in terms of the order parameter $\Delta m_{\vec{q}}$, with wave-vector modulation $\vec{q}$. They considered both Stoner particle-hole excitations ${ }^{10}$ and spin fluctuations ${ }^{11,13}$ generated from the collective behavior of the interacting electrons. ${ }^{11,14,15}$ Field-induced tricriticality occurs when the quadratic and quartic terms in $\Delta m_{\vec{q}}$ both equal zero. More recently, by analyzing a generic mean-field Hamiltonian describing a helical state in an applied field, Vareogiannis ${ }^{16}$ has shown how an itinerant electron system can undergo a first-order "spin-flip" transition. The AFM polarization is parallel to a weak applied field and flips perpendicularly only if the field exceeds a critical value. ${ }^{17}$

In this Rapid Communication we describe an $a b$ initio spin density functional theory (SDFT)-based "local moment" theory for AFM to suit real materials where both itinerant and local spin effects are at play. Slowly varying "local moments" can be identified from the complexity of the electronic behavior. We find that where magnetic anisotropy effects are small or neglected entirely, the local moments' AFM order can still undergo a first-order transition to a fan or FM state owing to the feedback between the local moment and itinerant aspects of the electronic structure. We test our theory against a detailed experimental case study of CoMnSi-based tricritical metamagnets and show how it provides quantitative materials-specific guidance for tuning metamagnetic and associated technological properties.

A generalization of SDFT ${ }^{18}$ describes the "local moment" picture of metallic magnets at finite temperature. ${ }^{19-22}$ Its basic 
premise is a time-scale separation between fast and slow electronic degrees of freedom so that local moments are set up with slowly varying orientations $\left\{\hat{e}_{i}\right\}$. The existence of these "disordered local moments" (DLMs) is established by the fast electronic motions and likewise their presence affects these motions. Moreover, the local moments' interactions depend on the type and extent of the long range magnetic order through the associated itinerant electronic structure. The DLM picture of the paramagnetic state maps to an Ising picture ${ }^{18}$ with, on average, one half of the moments oriented one way and the rest antiparallel. However, once the symmetry is broken so that there is a finite order parameter $\left\{\vec{m}_{i}\right\}$ profile, e.g., in a FM or AFM state and/or when an external magnetic field is applied, this simplicity is lost. Ensemble averages over the full range of noncollinear local moment orientational configurations $\left\{\hat{e}_{i}\right\}$ are needed to determine the system's magnetic properties realistically. ${ }^{22}$

We now develop the DLM theory for a magnetic material in an external magnetic field $\vec{B}$ at a temperature $T$. The probability that the system's local moments are configured according to $\left\{\hat{e}_{i}\right\}$ is $P_{i}\left(\left\{\hat{e}_{i}\right\}\right)=\exp \left[-\beta \Omega\left(\left\{\hat{e}_{i}\right\}, \vec{B}\right)\right] / Z$, where the partition function $Z=\prod_{j} \int d \hat{e}_{j} \exp \left[-\beta \Omega\left(\left\{\hat{e}_{i}\right\}, \vec{B}\right)\right], \beta=$ $\left(k_{B} T\right)^{-1}$, and the free energy $F=-k_{B} T \ln Z$. A "generalized" electronic grand potential $\Omega\left(\left\{\hat{e}_{i}\right\}, \vec{B}\right)$ is in principle available from SDFT, ${ }^{18}$ where the spin density is constrained to be orientated according to the local moment configuration $\left\{\hat{e}_{i}\right\}$. It thus plays the role of a local moment Hamiltonian but its genesis can give it a complicated form. Nonetheless, by expanding about a suitable reference "spin" Hamiltonian $\Omega_{0}\left\{\hat{e}_{i}\right\}=\sum_{i} \vec{h}_{i} \cdot \hat{e}_{i}$ and, using the Feynman inequality, ${ }^{23}$ we find a mean-field theoretical estimate of the free energy, ${ }^{18}$

$$
\begin{aligned}
F\left(\left\{\vec{m}_{i}\right\}, \vec{B}, T\right)= & \left\langle\Omega\left(\left\{\hat{e}_{i}\right\}, \vec{B}\right)\right\rangle_{\left\{\vec{m}_{i}\right\}} \\
& +k_{B} T \sum_{i} \int P_{i}\left(\hat{e}_{i}\right) \ln P_{i}\left(\hat{e}_{i}\right) d \hat{e}_{i} \\
& -\vec{B} \cdot \sum_{i} \mu_{i} \vec{m}_{i},
\end{aligned}
$$

where $P_{i}\left(\hat{e}_{i}\right)=\exp \left[-\beta \vec{h}_{i} \cdot \hat{e}_{i}\right] / \int \exp \left[-\beta \vec{h} \cdot \hat{e}_{i}\right] d \hat{e}_{i}$, so that the set of local order parameters, $\left\{\vec{m}_{i}\right\}=\left\{\int \hat{e}_{i} P_{i}\left(\hat{e}_{i}\right) d \hat{e}_{i}\right\}=$ $\left\{\left\langle\hat{e}_{i}\right\rangle\right\}$, each of which can take values between 0 and 1 . This DLM self-consistent mean-field theory of the statistical mechanics of the local moments can be seen as the natural counterpart of the DFT self-consistent description of the interacting electrons. The first term of Eq. (1) is the local moments' internal energy, i.e., the average of the electronic grand potential over local moment configurations consistent with the order parameter profile $\left\{\vec{m}_{i}\right\}$, the second is $(-T)$ multiplied by the local moments' entropy, and the last their interaction with a field $\vec{B}$. The sizes of the local moments $\left\{\mu_{i}\right\}$, are determined self-consistently ${ }^{18}$ via the generalized SDFT.

The Weiss field at a site $l$ is given by

$$
\vec{h}_{l}=-\frac{\partial\left\langle\Omega\left(\left\{\hat{e}_{i}\right\}, \vec{B}\right)\right\rangle_{\left\{\vec{m}_{i}\right\}}}{\partial \vec{m}_{l}} .
$$

To capture the itinerant electronic component of the problem coming from the overall spin polarization of the electronic structure, we approximate the $\vec{h}_{l}$ via an expansion about a uniform $\vec{m}=\frac{1}{N} \sum_{i} \vec{m}_{i}$, i.e.,

$$
\begin{aligned}
\vec{h}_{l} & \approx-\left.\frac{\partial\langle\Omega\rangle}{\partial \vec{m}_{l}}\right|_{\vec{m}}-\left.\sum_{j} \frac{\partial^{2}\langle\Omega\rangle}{\partial \vec{m}_{l} \partial \vec{m}_{j}}\right|_{\vec{m}} \cdot\left(\vec{m}_{j}-\vec{m}\right) \\
& =\vec{h}(\vec{m})+\sum_{j} \tilde{S}_{l, j}^{(2)}(\vec{m}) \cdot\left(\vec{m}_{j}-\vec{m}\right) .
\end{aligned}
$$

$\tilde{S}_{i, j}^{(2)}(\vec{m})$ is the direct correlation function and crucially describes effective interactions between the local moments that depend on the magnitude of $\vec{m}$. A solution of Eqs. (1)-(3) at a fixed $T$ and applied field $\vec{B}$ minimizes the free energy $F$, Eq. (1). Several solutions, $\left\{\vec{m}_{l}^{(1)}\right\},\left\{\vec{m}_{l}^{(2)}\right\}, \ldots$, may be found and the one with the lowest $F$ describes the system's equilibrium state $\left\{\vec{m}_{l}\right\}_{\text {Equil. }}$. Hence metamagnetic transitions can be tracked as functions of $T$ and $\vec{B}$ - for a given $T$ the solutions for increasing values of $B$ can show a transition from, say, an AFM to a high magnetization state at a critical field $B_{c}$ [e.g., see Fig. 1(b)]. The material's spin-polarized electronic structure also depends on $B$ and $T$ and the state of magnetic order.

In order to elucidate the main aspects of the general framework laid out in Eqs. (1)-(3) for a putative helical metal, we assume temporarily that magnetic anisotropy effects are small and can be neglected. Without a magnetic field $\vec{B}$, the solution $\left\{\vec{m}_{l}\right\}$ which produces the lowest free energy is $\vec{m}_{i}=\Delta m_{\vec{q}}\left[\cos \left(\vec{q} \cdot \vec{R}_{i}\right) \hat{x}+\sin \left(\vec{q} \cdot \vec{R}_{i}\right) \hat{y}\right]$. The helical axis $\hat{z}$ has no preferred direction in the crystal lattice. The order parameter $\Delta m_{\vec{q}}$ increases from 0 to 1 as $T$ drops from $T_{N}$ to $0 \mathrm{~K}$. On applying $\vec{B}$, defining, say, the $X$ axis of our coordinate frame, we find numerical solutions of the meanfield equations (1)-(3) of (i) distorted helical form, $\vec{m}_{i}=(m+$ $\left.\Delta m_{\vec{q}}\right) \cos \left(\vec{q} \cdot \vec{R}_{i}\right) \hat{x}+\Delta m_{\vec{q}} \sin \left(\vec{q} \cdot \vec{R}_{i}\right) \hat{y}$, (ii) a conical helix, $\vec{m}_{i}=m \hat{x}+\Delta m_{\vec{q}}\left[\cos \left(\vec{q} \cdot \vec{R}_{i}\right) \hat{y}+\sin \left(\vec{q} \cdot \vec{R}_{i}\right) \hat{z}\right]$, (iii) a fan state, $\vec{m}_{i}=m \hat{x}+\Delta m_{\vec{q}} \cos \left(\vec{q} \cdot \vec{R}_{i}\right) \hat{y}$, and (iv) a high magnetization state with $\Delta m_{\vec{q}}=0$. The free energies, functions of $m, \Delta m_{\vec{q}}$, $\vec{B}$, and $T$, are $F_{\mathrm{dh}}, F_{\mathrm{ch}}, F_{\mathrm{fan}}$ and $F_{\mathrm{FM}}$, respectively, and we search for the lowest one at a given $\vec{B}$ and $T$. Consequently the relative difference between $F_{\mathrm{dh}}$ and any of the others determines whether or not there is a first-order metamagnetic transition and also the $T$ dependence of the critical field $\vec{B}_{c}$.

The local moment interactions $\tilde{S}_{i, j}^{(2)}(\vec{m})$ of Eq. (3) set up the Weiss field and, along with the uniform component $\vec{h}(\vec{m}),{ }^{22}$ are the key elements of our theory. We determine these quantities using relativistic, spin-polarized, multiple scattering [Korringa-Kohn-Rostoker (KKR)] theory and the coherent potential approximation $(\mathrm{CPA}) .^{22,24,25} \mathrm{We}$ account for the variation of $\tilde{S}_{i, j}^{(2)}(\vec{m})$ with increasing $\vec{m}$ driven by spin polarization of the itinerant electrons mediating the local moments' interactions. ${ }^{26,27}$ Technical details on the calculations are found in the Supplemental Material. ${ }^{28}$

Our previous experimental investigations revealed a class of magnetic materials based on the orthorhombic CoMnSi metal to be an ideal testing ground for the theory. ${ }^{29,30}$ Those extensive magnetic and structural studies considered both the composition-dependent metamagnetism and pronounced magnetoelasticity in CoMnSi, including a characterization of 

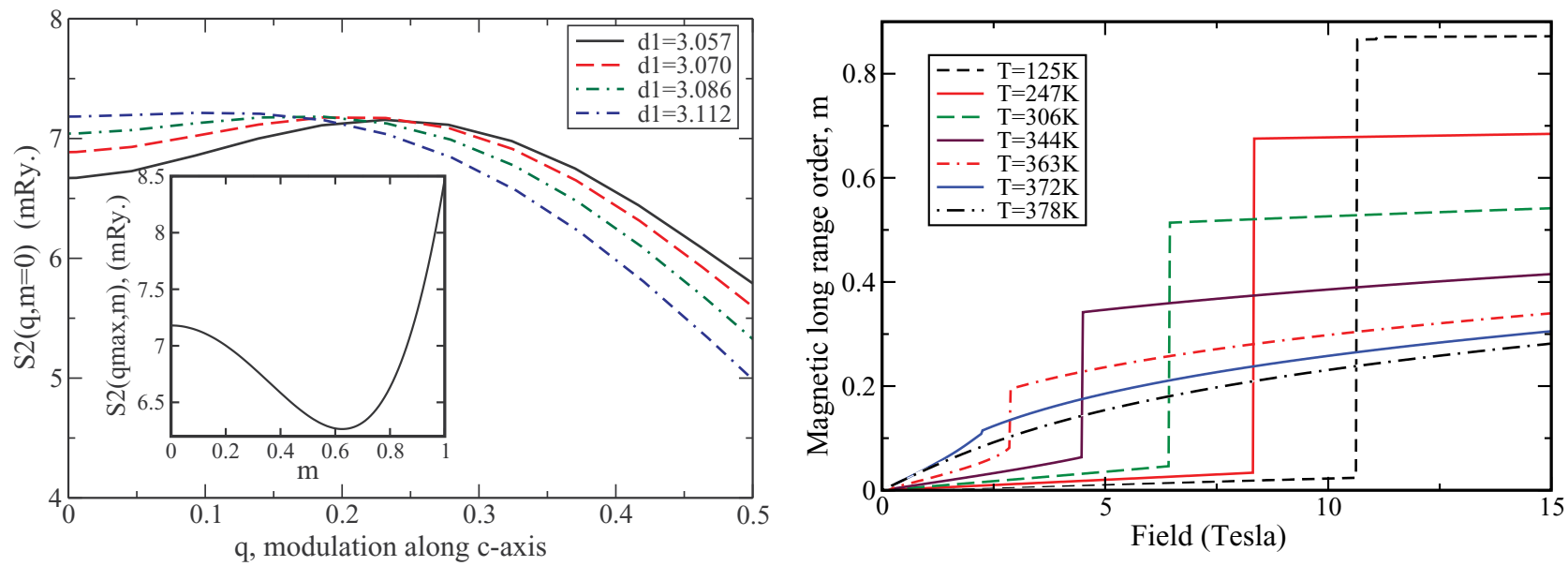

FIG. 1. (Color online) (a) The Mn local moment interactions $S^{(2)}(\vec{q}, \vec{m}=0)$ for CoMnSi with structures measured in neutron diffraction experiments labeled by the Mn-Mn spacing $d_{1}$ in $\AA$. The inset shows $S^{(2)}\left(\vec{q}_{\max }, \vec{m}\right)$ vs order parameter $m$ for $d_{1}=3.07 \AA$. (b) $m$ vs applied field for several temperatures for a fixed lattice structure, in which $d_{1}=3.07 \AA$. A tricritical point is indicated at $372 \mathrm{~K}, 2 \mathrm{~T}$.

the anomalous temperature variation of structural parameters in zero magnetic field. CoMnSi orders into a noncollinear, helical AFM state in zero magnetic field at $T_{N} \approx 380 \mathrm{~K}$. In an applied field $\vec{B}$, this transition becomes a metamagnetic one to a high magnetization state at $T_{t}$. As $B$ is increased, $T_{t}$ decreases before going through a tricritical point at around $2 \mathrm{~T}$, where enhanced magnetocaloric and magnetostructural effects are observed. Typical of many useful magnetic metals, CoMnSi has magnetism with both localized and itinerant electron spin attributes. ${ }^{17}$ Localized magnetic moments are identified with the Mn sites whereas the magnetism associated with the Co sites is reflected in the long range spin polarization of the electronic structure. Our measurements found that the magnetism of CoMnSi is dominated by the behavior of the Mn moments. Their interactions, however, are delicately poised depending on the spacing between them ${ }^{31}$ and their compositional environment.

We start with DFT-DLM calculations ${ }^{32-34}$ for the paramagnetic $(m=0)$ state of CoMnSi. Local moments of magnitude $\mu \approx 3.0 \mu_{B}$ establish on the Mn sites which are very close to the magnetization per Mn site we, and another study, ${ }^{35}$ found in calculations of CoMnSi in a FM state $(m=1)$, showing that the Mn local moments' sizes are rather insensitive to the orientations of moments surrounding them. We thus use the "frozen potential" approximation ${ }^{24,36}$ to study CoMnSi for $\left\{P_{i}\left(\hat{e}_{i}\right)\right\}$, producing magnetic order parameters $\vec{m}_{i}$, each ranging between 0 and 1 . For a finite long range order parameter $m \neq 0$, we consider a probability distribution on a fine grid of local moment orientations. ${ }^{22,24}$ No local moment forms on the cobalt sites in the DLM paramagnetic state (nor in our model of a commensurate approximation to the helical AFM state ${ }^{29}$ found in experiment) whereas for finite $m$, a small magnetization associated with each Co site is induced by the Mn moments lining up and the consequent overall spin polarization of the electronic structure. For the FM state, $m=1$, this is $\approx 0.6 \mu_{B}$ per Co site. $^{29,35}$

The onset of magnetic order is found from examining $\tilde{S}_{i, j}^{(2)}(\vec{m})$ for the paramagnetic state, $m=0$. By calculating the lattice Fourier transform $\tilde{S}^{(2)}(\vec{q}, 0)$ and finding the wave vector $\vec{q}_{\max }$ where it is greatest, we find the magnetically ordered state that the system forms below our mean-field theory estimate of $T_{N}=S^{(2)}\left(\vec{q}_{\max }, 0\right) / 3 k_{B}$. If $q_{\max }=0$, a FM state is indicated, whereas $q_{\max } \neq 0$ indicates an AFM state. Figure 1(a) shows $S^{(2)}(\vec{q}, 0)$ using our structural data from neutron diffraction ${ }^{29}$ which show the Mn-Mn distances $d_{1}$ to vary by more than $2 \%$ over the temperature range $100-400 \mathrm{~K}$. Our calculations show that CoMnSi should order into an incommensurate helical AFM state along the $C$ axis, set by the orthorhombic crystal structure, at $T_{N} \approx 400 \mathrm{~K}$, in good agreement with experiment. ${ }^{29,30}$ Since our calculations include spin-orbit coupling effects we checked that magnetic anisotropy effects are indeed small for this material (see Ref. 28), as seen in measurement. ${ }^{37}$

CoMnSi is near a FM instability, as shown by the values of $S^{(2)}(\vec{q}, 0)$ in Fig. 1(a) at $\vec{q}=0$ and $\vec{q}_{\text {max }}$ differing only slightly, a convenient signature for a potentially useful metamagnet. The tendency to order ferromagnetically takes over from the incommensurate ordering propensity as $d_{1}$ is increased, which experiment also shows to happen with increasing temperature. When the bath of electrons in which the Mn local moments sit becomes spin polarized as a magnetic field is applied and $m$ increases, we find that the magnetic interactions $\tilde{S}^{(2)}(\vec{q}, \vec{m})$ weaken significantly, as shown by the example of the inset to Fig. 1(a). ${ }^{28}$ This weakening promotes the stability of the distorted helical state over the others when the magnetic field is applied..$^{35}$ There can therefore be a first-order transition to a fan, conical helix, or high magnetization state at critical field $B_{c}$ despite the absence of magnetic anisotropy. Figure 1(b) illustrates this effect, and shows our solutions of the mean-field theory equations (1)-(3) for one example. We point out that there are no adjustable parameters for these curves which show a transition from a distorted helical state ( $\operatorname{small} m, B$ ) to a high magnetization state above a critical field $B_{c}$. The transition is first order up to a tricritical temperature of $372 \mathrm{~K}$ and second order thereafter.

Figure 2 shows the effect on $B_{c}(T)$ of either changing $\mathrm{Mn}$ nearest-neighbor separations $d_{1}$ [Fig. 2(a)] or varying composition slightly away from CoMnSi [Fig. 2(b)]. Tricritical 

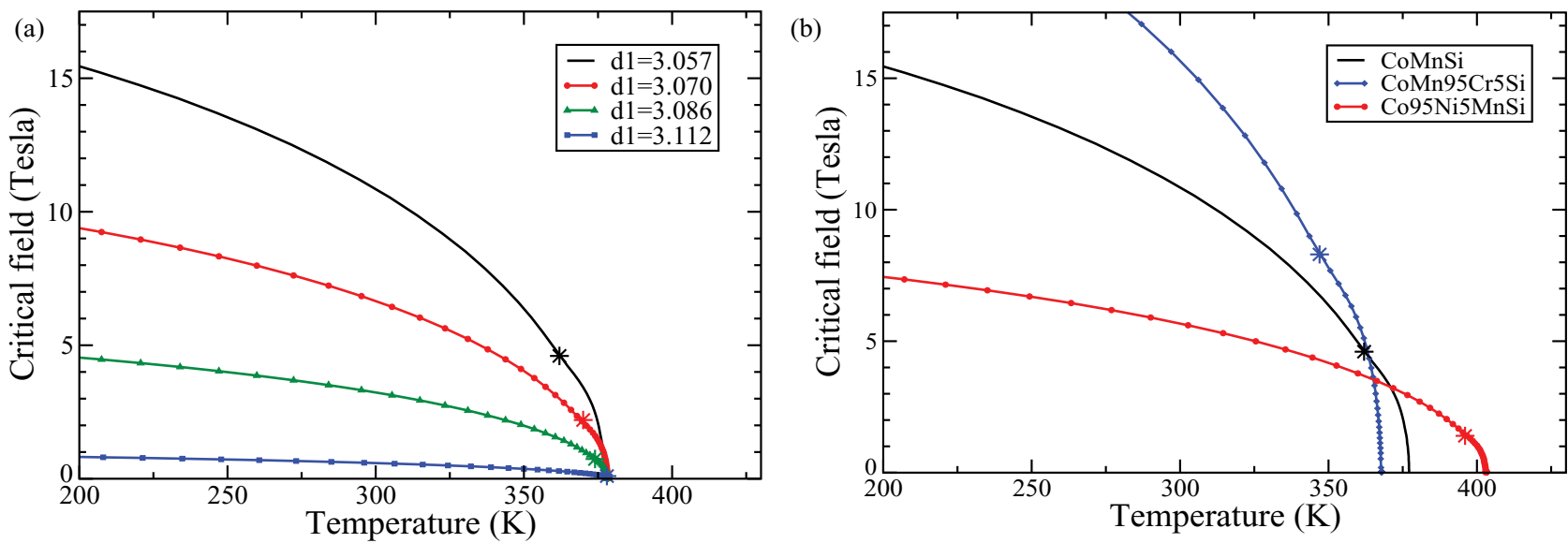

FIG. 2. (Color online) (a) The critical field $B_{c}$ for a transition between a helical AFM and a high magnetization state vs $T$ for CoMnSi for the four structures used in Fig. 1 and (b) for $\mathrm{Co}_{0.95} \mathrm{Ni}_{0.05} \mathrm{MnSi}$ and $\mathrm{CoMn}_{0.95} \mathrm{Cr}_{0.05} \mathrm{Si}$, with $d_{1}=3.057 \AA$ (tricritical points denoted by asterisks).

points are denoted by asterisks. If the itinerant electron effect which gives $\tilde{S}^{(2)}(\vec{q}, \vec{m})$ its $\vec{m}$ dependence is neglected and only the effects of a weak on-site magnetocrystalline anisotropy are included, ${ }^{28}$ we find the values of $B_{c}$ to be two orders of magnitude smaller. $B_{c}$ increases sharply as $d_{1}$ decreases, in line with the measured pressure dependence of $B_{c}$ of CoMnSi, ${ }^{38}$ and is very sensitive to compositional dopingthe AFM tendency strengthens as electrons are removed $\left(\mathrm{CoMn}_{0.95} \mathrm{Cr}_{0.05} \mathrm{Si}\right)$ so that $B_{c}$ increases, whereas it weakens when electrons are added $\left(\mathrm{Co}_{0.95} \mathrm{Ni}_{0.05} \mathrm{MnSi}\right)$.

To test the theory presented here we have used structural data taken in zero magnetic field ${ }^{30}$ to predict the $T$ dependence of $B_{c}$ in three CoMnSi-based compounds, comparing our findings with the experimental magnetic phase diagram extracted from magnetization isotherms. ${ }^{30}$ The lower panel, Fig. 3(b), shows our theoretical estimates of the critical fields.



FIG. 3. (Color online) (a) Experimental $B_{c}(T)$ for CoMnSi, $\mathrm{Co}_{0.95} \mathrm{Ni}_{0.05} \mathrm{MnSi}$, and $\mathrm{CoMn}_{0.98} \mathrm{Cr}_{0.02} \mathrm{Si}$ (Ref. 30). (b) Theoretical $B_{c}(T)$ for the same three systems, modeled using the lattice parameters found from neutron diffraction (Ref. 30) in zero field. The $B_{c}(T)$ of the Ni- and Cr-doped systems modeled using the CoMnSi structural data is also shown (dashed lines) to highlight the relative importance of accurate structural data.
The magnitudes and trends are given very well by the theory. The increase of $\mathrm{Mn}-\mathrm{Mn}$ separation $d_{1}$ with temperature is responsible for the concave appearance of $B_{c}(T)$ in CoMnSi. The use of correct structural data is very important to model the Ni-doped material. If we instead use CoMnSi data to model this compound, we find much higher $B_{c}(T)$ values. This material is closer to ferromagnetism, as observed experimentally. ${ }^{30}$

Detailed theoretical models are needed to use in concert with experimental studies if the behavior of highly sensitive magnetic materials is to be analyzed and tuned. We have discussed one such model here and showed how the temperature and field dependence of the metamagnetic transition of an antiferromagnetic metal can be affected by varying composition and atomic spacing. Useful magnetic metals typically have large magnetic moments which are established locally. The disordered local moment model works well for these $\mathrm{e}^{22,39,40}$ and we have set out a theory for metamagnetic transitions based on this picture. An important aspect for magnetic material design is to distinguish first- and second-order magnetic transitions and to get some control of the location of precious tricritical points. Our CoMnSi case study reveals how decreasing the Mn-Mn separation $d_{1}$ or reducing the number of electrons by compositional doping increases the critical field. One notable feature in our modeling is the dependence of the interaction between the local moments upon the extent of long range magnetic order. This comes from the change in the behavior of the itinerant electrons that mediate these interactions and produces a mechanism for first-order "spin-flip" transitions, even for cases where there is little magnetic anisotropy. This aspect has an important role in the analysis and design of adaptive, magnetic metals.

We acknowledge financial support from the EPSRC (UK) (J.B.S., J.P.), a FCT Portugal Ph.D. Grant No. SFRH/BD/35738/2007 (M.d.S.D.), the Royal Society (K.G.S.), and EPSRC Grants No. EP/G060940/1 and No. EP/E016243/1 (Z.G.). K.G.S. thanks M. Avdeev and J. Bechhöfer for useful discussions. 
${ }^{1}$ E. Stryjewski and N. Giordano, Adv. Phys. 26, 487 (1977).

${ }^{2}$ I. S. Jacobs and P. E. Lawrence, Phys. Rev. 164, 866 (1967).

${ }^{3}$ I. S. Jacobs and P. E. Lawrence, J. Appl. Phys. 35, 996 (1964).

${ }^{4}$ K. G. Sandeman, Scr. Mater. 67, 566 (2012).

${ }^{5}$ M. P. Annaorazov, K. A. Asatryan, G. Myalikgulyev, S. A. Nikitin, A. M. Tishin, and A. L. Tyurin, Cryogenics 32, 867 (1992).

${ }^{6}$ P. M. Chaikin and T. C. Lubensky, Principles of Condensed Matter Physics (Cambridge University Press, Cambridge, UK, 2000).

${ }^{7}$ J. Liu, J. D. Moore, K. P. Skokov, M.Krautz, K. Lowe, A. Barcza, M. Katter, and O. Gutfleisch, Scr. Mater. 67, 584 (2012).

${ }^{8}$ N. H. Dung, Z. Q. Ou, L. Caron, L. Zhang, D. T. Cam Thanh, G. A. de Wijs, R. A. de Groot, K. H. J. Buschow, and E. Brueck, Adv. Energy Mater. 1, 1215 (2011).

${ }^{9}$ T. Nagamiya, K. Nagata, and Y. Kitano, Prog. Theor. Phys. 27, 1253 (1962).

${ }^{10}$ E. P. Wohlfarth and P. Rhodes, Philos. Mag. 7, 1817 (1962).

${ }^{11}$ T. Moriya and K. Usami, Solid State Commun. 23, 935 (1977).

${ }^{12}$ M. Shimuzu, J. Phys. 43, 155 (1982).

${ }^{13}$ H. Yamada and T. Goto, Phys. Rev. B 68, 184417 (2003).

${ }^{14}$ Electron Correlations and Magnetism in Narrow-Band Systems, edited by T. Moriya (Springer, New York, 1981).

${ }^{15} \mathrm{~J}$. Kübler, Theory of Itinerant Electron Magnetism (Clarendon, Oxford, UK, 2000).

${ }^{16}$ G. Varelogiannis, Phys. Rev. Lett. 91, 117201 (2003).

${ }^{17}$ K. Irisawa, A. Fujita, K. Fukamichi, M. Yamada, H. Mitamura, T. Goto, and K. Koyama, Phys. Rev. B 70, 214405 (2004).

${ }^{18}$ B. L. Gyorffy, A. J. Pindor, J. Staunton, G. M. Stocks, and H. Winter, J. Phys. F 15, 1337 (1985).

${ }^{19}$ J. Hubbard, Phys. Rev. B 20, 4584 (1979).

${ }^{20}$ H. Hasegawa, J. Phys. Soc. Jpn. 46, 1504 (1979).

${ }^{21}$ J. B. Staunton and B. L. Gyorffy, Phys. Rev. Lett. 69, 371 (1992).

${ }^{22}$ J. B. Staunton, S. Ostanin, S. S. A. Razee, B. L. Gyorffy, L. Szunyogh, B. Ginatempo, and E. Bruno, Phys. Rev. Lett. 93, 257204 (2004).

${ }^{23}$ R. P. Feynman, Phys. Rev. 97, 660 (1955).

${ }^{24}$ J. B. Staunton, L. Szunyogh, A. Buruzs, B. L. Gyorffy, S. Ostanin, and L. Udvardi, Phys. Rev. B 74, 144411 (2006).
${ }^{25}$ M. dos Santos Dias, J. B. Staunton, A. Deak, and L. Szunyogh, Phys. Rev. B 83, 054435 (2011).

${ }^{26}$ L. M. Sandratskii, R. Singer, and E. Sasioglu, Phys. Rev. B 76, 184406 (2007)

${ }^{27}$ S. Polesya, S. Mankovsky, O. Sipr, W. Meindl, C. Strunk, and H. Ebert, Phys. Rev. B 82, 214409 (2010).

${ }^{28}$ See Supplemental Material at http://link.aps.org/supplemental/ 10.1103/PhysRevB.87.060404 for (i) the calculations of Weiss fields (Eq. 2) and local moments interactions (Eq. 3) with further examples and a comment on magnetic anisotropic effects and (ii) structural and compositional effects on critical fields relevant to Fig. 3.

${ }^{29}$ A. Barcza, Z. Gercsi, K. S. Knight, and K. G. Sandeman, Phys. Rev. Lett. 104, 247202 (2010).

${ }^{30}$ A. Barcza, Z. Gercsi, H. Michor, K. Suzuki, W. Kockelmann, K. S. Knight, and K. G. Sandeman, Phys. Rev. B 87, 064410 (2013).

${ }^{31}$ Z. Gercsi, K. Hono, and K. G. Sandeman, Phys. Rev. B 83, 174403 (2011).

${ }^{32}$ G. M. Stocks, W. M. Temmerman, and B. L. Gyorffy, Phys. Rev. Lett. 41, 339 (1978).

${ }^{33}$ D. D. Johnson, D. M. Nicholson, F. J. Pinski, B. L. Gyorffy, and G. M. Stocks, Phys. Rev. Lett. 56, 2088 (1986).

${ }^{34}$ H. Ebert, D. Koedderitzsch, and J. Minár, Rep. Prog. Phys. 74, 096501 (2011).

${ }^{35}$ V. I. Valkov, A. V. Golovchan, E. A. Dvornikov, and B. M. Todris, Low Temp. Phys. 36, 1064 (2010).

${ }^{36}$ J. Harris, Phys. Rev. B 31, 1770 (1985).

${ }^{37}$ K. Morrison, Y. Miyoshi, J. D. Moore, A. Barcza, K. G. Sandeman, A. D. Caplin, and L. F. Cohen, Phys. Rev. B 78, 134418 (2008).

${ }^{38}$ Yu. D. Zavorotnev, L. T. Medvedeva, B. M. Todris, E. A. Dvornikov, and O. Yu. Popova, J. Magn. Magn. Mater. 323, 2808 (2011).

${ }^{39}$ I. D. Hughes, M. Daene, A. Ernst, W. Hergert, M. Lueders, J. Poulter, J. B.Staunton, A. Svane, Z. Szotek, and W. M. Temmerman, Nature (London) 446, 650 (2007).

${ }^{40}$ I. D. Hughes, M. Däne, A. Ernst, W. Hergert, M. Lüders, J. B. Staunton, Z. Szotek, and W. M. Temmerman, New J. Phys. 10, 063010 (2008) 